\title{
MicroRNA-92a promotes epithelial-mesenchymal transition through activation of PTEN/PI3K/AKT signaling pathway in non-small cell lung cancer metastasis
}

\author{
CHAOJING LU ${ }^{1 *}$, ZHENGXIANG SHAN $^{2 *}$, JIANG HONG $^{1}$ and LIXIN YANG ${ }^{1}$ \\ ${ }^{1}$ Department of Thoracic Surgery, Changhai Hospital, Second Military Medical University; \\ ${ }^{2}$ Department of Thoracic Surgery, Eastern Hepatobiliary Surgery Hospital, \\ Second Military Medical University, Shanghai, P.R. China
}

Received February 10, 2017; Accepted April 18, 2017

DOI: 10.3892/ijo.2017.3999

\begin{abstract}
MicroRNAs (miRNAs) have important roles in various cancers, including non-small cell lung cancer (NSCLC). Although several miRNAs have reported to be involved in the development of NSCLC, understanding the regulatory roles of other miRNAs in NSCLC is essential. Therefore, the aim of the current study was to explore the roles and mechanisms of screened miRNAs in NSCLC. First, the differentially expressed miRNAs that were screened based on GSE29248 microarray data retrieved from Gene Expression Omnibus (GEO). The expression of miR-92a, acted as an oncogene in many cancers, was validated using quantitative real-time PCR (qRT-PCR), and then its association with overall survival was analyzed. The efficacy of miR-92a to promote cell proliferation, invasion and metastasis was evaluated in vitro, and in vivo. Then, the role of miR-92a in epithelialmesenchymal transition (EMT), a key step of the progression of tumor cell metastasis, was investigated in NSCLC cells. The association of miR-92a and its downstream target was investigated in both cell line and clinical specimens. Furthermore, gain- and loss-of-function studies of the phosphatase and tensin homolog (PTEN) were performed to assess whether the effect of miR-92a promoted growth and metastasis of NSCLC cells were via targeting PTEN. Our results showed that miR-92a was significantly upregulated in NSCLC tissues and NSCLC cell lines, and was positively associated with poor prognosis of NSCLC patients. The overexpression of miR-92a enhanced EMT-relatived protein levels, promoted NSCLC cell migration and invasion in vitro, and increased tumor growth
\end{abstract}

Correspondence to: Dr Lixin Yang, Department of Thoracic Surgery, Changhai Hospital, Second Military Medical University, 168 Changhai Road, Shanghai 200433, P.R. China

E-mail: yanglixin_76@126.com

*Contributed equally

Key words: non-small cell lung cancer, miR-92a, metastasis, epithelial-mesenchymal transition, PTEN/PI3K/AKT in vivo. Bioinformatic prediction and function assay suggested that PTEN, a negative regulator of PI3K/AKT pathway, was a direct target of miR-92a. It was found that PTEN expression was inversely correlated with miR-92a in NSCLC tissues. In addition, miR-92a could activate the PI3K/AKT pathway by inhibiting PTEN expression. Notably, Transwell and wound healing assays demonstrated that altering PTEN expression abrogated the promotive effects of miR-92a on NSCLC cell migration and invasion. Taken together, these results demonstrated that miR-92a induced EMT and regulated cell migration and invasion in the NSCLC cells through regulating PI3K/AKT signaling pathway by targeting PTEN, indicating that miR-92a may be an attractive target and prognostic marker for NSCLC.

\section{Introduction}

Non-small cell lung cancer (NSCLC) is a main type of lung cancer, which accounts for approximately $85 \%$ of all lung cancer patients in China (1). Despite significant improvement in surveillance and targeted therapy, the 5-year survival rate of patients after curative resection is very low, which is reported to be only $30-60 \%$ mainly because of tumor metastasis (2). Therefore, understanding the potential molecular mechanism involved in NSCLC metastasis may contribute to improve diagnosis and treatment of NSCLC.

MicroRNA (miRNA) is a class of small non-proteincoding RNAs which is composed of 21-23 bases that negatively regulate protein-coding gene expression and/or repress mRNA translation by binding to the 3 '-untranslated region (3'-UTR) $(3,4)$. Previous studies have reported that miRNAs play pivotal roles in a wide range of cellular processes such as proliferation, cycle, differentiation, apoptosis and metastasis (5). The miRNAs have been confirmed to function either as oncomiRs or tumor suppressors in lung cancer (6-8). For example, miR-383 is significantly downregulated in NSCLC cell lines and NSCLC carcinomas tissues, which is a functional tumor suppressor in NSCLC (9). Zeng et al reported that ectopic expression of miR-205 in NSCLC cells suppressed cellular viability and proliferation, accelerated the cell cycle, and promoted tumor growth of lung carcinoma xenografts in 
nude mice (10). Thus, identification of tumor-suppressive or oncogenic miRNAs may be the first step in construction of a new treatment strategy for NSCLC.

Epithelial-mesenchymal transition (EMT) is an intricate process by which epithelial cells lose their epithelial characteristics and adopt a mesenchymal-like phenotype (11). Increasing evidence suggest that EMT plays a key role in tumor progression and metastasis $(12,13)$. The molecular mechanisms of EMT in tumor metastasis are very complex. Multiple molecules are known to regulate EMT, including miRNAs (14-16). Some miRNAs (for example, the miR-200 family and miR-145) have been shown to regulate EMT in cancers (17-19). However, studies of the roles of other miRNAs in the regulation of EMT are limited.

In this study, we found that upregulation of miR-92a could promote cell invasion in vitro and tumor growth in vivo in NSCLC. Furthermore, we demonstrated that miR-92a promoted the phosphoinositide 3-kinase (PI3K)/AKT signaling pathway and induced EMT by targeting PTEN. The findings in the present study revealed that miR-92a could induce EMT phenotype via regulating PTEN-mediated AKT pathway, influencing cancer progression, invasion and metastasis.

\section{Materials and methods}

Cell culture and tissue samples. One human bronchial epithelial cell line 16HBE and four NSCLC cell lines (A549, H358, SPC-A1 and H1299) were used in this study, all cell lines were obtained from the Cell Culture Center of the Shanghai Institute (Shanghai, China) and cultured in DMEM (Dulbecco's minimum essential medium) containing 10\% fetal bovine serum (Gibco, Grand Island, NY, USA) at $37^{\circ} \mathrm{C}$ in a humidified incubator with $5 \% \mathrm{CO}_{2}$.

Fifty pairs of NSCLC tissues were obtained randomly from patients who underwent surgical resection at Changhai Hospital, Second Military Medical University between April 2014 and August 2015. None of the patients received chemotherapy or radiotherapy before surgery. All human materials were obtained with informed consent from patients and were approved by the ethics committees of the Second Military Medical University. The tissues were stored at $-80^{\circ} \mathrm{C}$.

MicroRNA expression profile data from Gene Expression Omnibus (GEO). We downloaded the microRNA data (accession number: GSE29248) from GEO databases in NCBI (http://www.ncbi.nlm.nih.gov/geo/). The microarray data were generated using Affymetrix U133A/B and Plus 2.0 platforms. After inter array quantile normalization, the expression levels of 50 miRNAs were visually generated as a heat map using GeneSpring GX, version 7.3 statistical software.

Transfection. Oligonucleotides including miR-92a mimics, miR-92a inhibitor, PTEN siRNA and their negative control (NC) were purchased from Shanghai GenePharma Co. Ltd. A549 and H358 cells were plated in 6-well plates and transfected with $50 \mathrm{nM}$ miRNAs or PTEN siRNA with Lipofectamine RNAiMAX (Invitrogen, Carlsbad, CA, USA) according to the manufacturer's protocol. The coding sequences of PTEN were amplified by PCR and inserted into pcDNA3.0 vector to construct the PTEN overexpression vector. The transfection of plasmids was conducted using Lipofectamine 2000 (Invitrogen). All the transfections were repeated more than three times independently.

Quantitative reverse transcription-polymerase chain reaction $(q R T-P C R)$. Total RNA of the cultured cells and the tissues was extracted using TRIzol (Invitrogen) according to the manufacturer's instructions. Total RNA from each sample was reverse-transcribed to cDNA using the PrimeScript RT reagent kit (Takara, Tokyo, Japan). The procedure for qRT-PCR was previously described (20). To normalize the data for quantifcation of mRNA and miRNAs, the GAPDH and U6 (Applied Biosystems) were used as internal standards for miRNA and mRNA, respectively. The sequences of the PCR primers were as follows: PTEN forward, 5'-CGGCAGCATCAAATGTT TCAG-3' and reverse 5'-AACTGGCAGGTAGAAGGCAA CTC-3'; GAPDH forward, 5'-CTCCTCCTGTTCGACA GTCAGC-3', and reverse 5'-CCCAATACGACCAAATCC GTT-3'. miR-92a forward 5'-CTGTCCTGTTATTGAGCACT GGTCTATGG-3' and reverse 5'-AAGACATTAGTAACCCA CCCCCATTCC-3'; U6 forward, 5'-CTCGCTTCGGCAGC ACA-3' and reverse, 5'-AACGCTTCACGAATTTGCGT-3'.

Cell viability assay. The effect of miR-92a on the viability of A549 and H358 cells was examined using the MTT assay. Briefly, A549 and H358 cells were seeded in a 96-well plate ( $8 \times 10^{3}$ cells/well) in $100 \mu 1$ growth medium after transfection. After $48 \mathrm{~h}$ incubation at $37^{\circ} \mathrm{C}, 20 \mu \mathrm{l}$ of MTT solution $(5 \mathrm{mg} / \mathrm{ml})$ was added to each well, and the cells were continuously incubated for $4 \mathrm{~h}$ before $200 \mu \mathrm{l}$ DMSO was added. The absorbance was read with a microplate reader (BioTek, Winooski, VT, USA) at $570 \mathrm{~nm}$ according to the manufacturer's instructions.

Apoptosis assay. The effect of miR-92a on the apoptosis of A549 and H358 cells was examined using an Annexin V-FITC apoptosis detection kit (Life Technologies). After $24 \mathrm{~h}$ posttransfection, A549 and H358 cells were collected, centrifuged and resuspended in $100 \mu 1$ binding buffer. Propidium iodide (PI) $(3 \mu \mathrm{l})$ and Annexin V-FITC (6 $\mu \mathrm{l})$ were added to each $100 \mu \mathrm{l}$ sample and incubated for $15 \mathrm{~min}$ at room temperature in the dark. The samples were analyzed on a FACScalibur flow cytometer to determine rate of apoptosis.

Cell invasion assays. The effect of miR-92a on cell invasion was evaluated using Transwell chamber assay (BD Biosciences, Bedford, MA, USA) according to the manufacturer's instructions. Briefly, $5 \times 10^{4}$ A549 and H358 cells were seeded on the top chamber coated with Matrigel after transfection. The bottom chambers were filled with $20 \%$ FBS in DMEM. After incubated at $37^{\circ} \mathrm{C}$ for $48 \mathrm{~h}$, the cells adherent to the upper surface of the filter were removed using a cotton swab. Then the migration cells were stained with crystal violet after fixed with $4 \%$ paraformaldehyde for $20 \mathrm{~min}$, and the migrated cells were counted by averaging the total number of cells from triplicate determinations.

Wound healing assay. The effect of miR-92a on cell migration was evaluated with wound healing assays. Cells were plated in 6 -well plates at $8 \times 10^{5}$ cells/well, and after $48 \mathrm{~h}$ of transfection, 
A

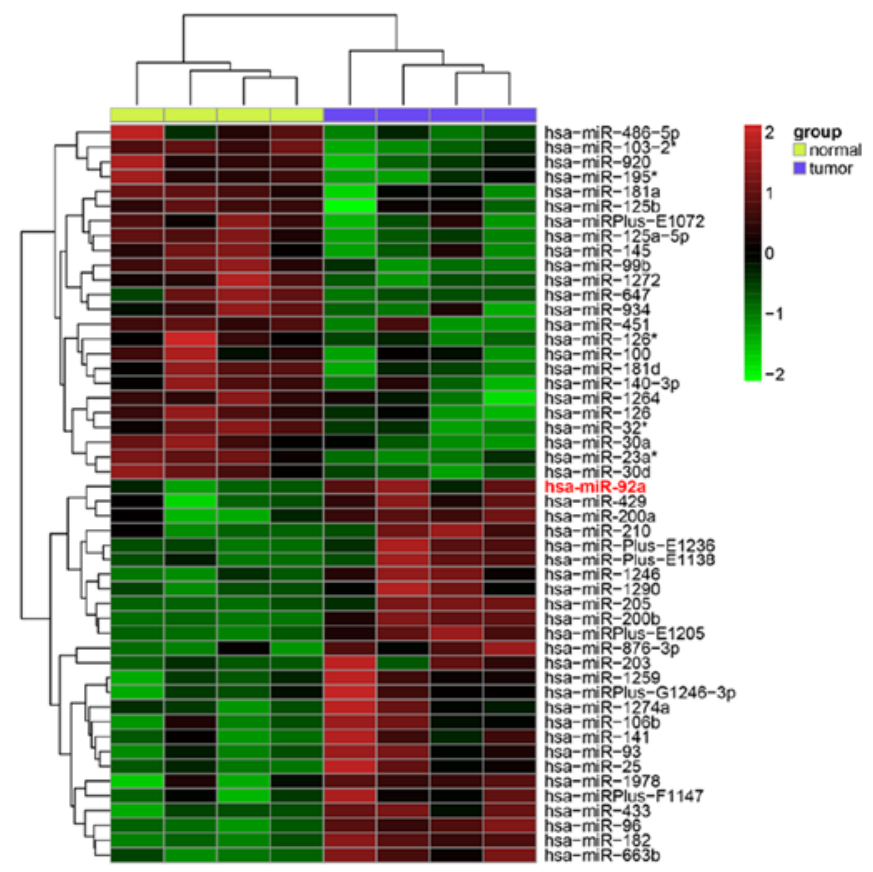

B

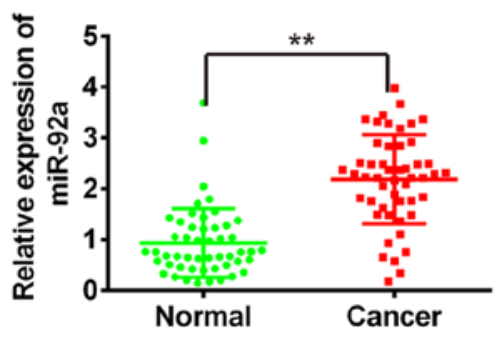

C

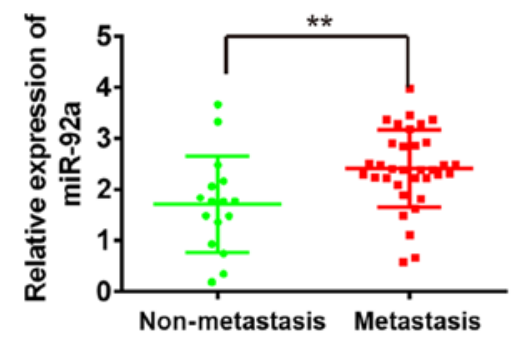

D

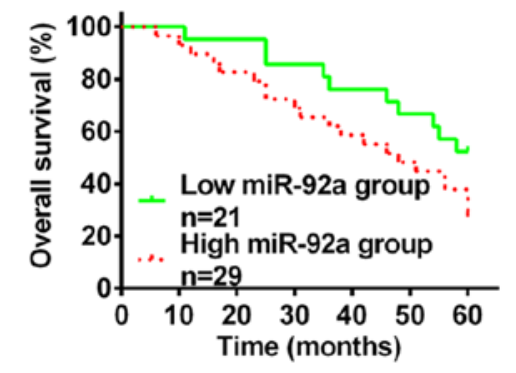

E

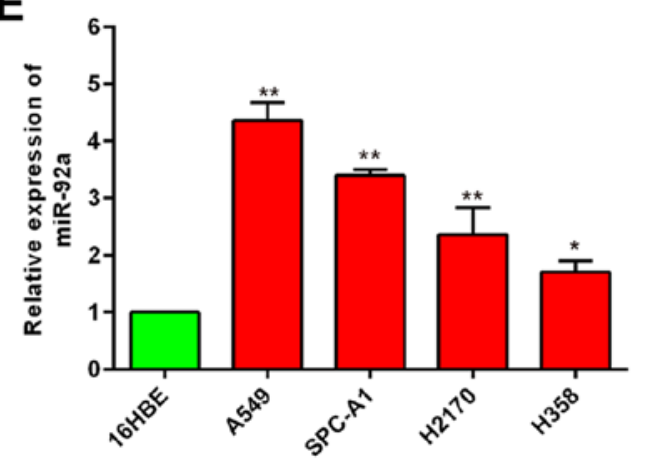

Figure 1. miR-92a is upregulated in NSCLC tissues and cell lines. (A) Differentially expressed miRNAs were analyzed between NSCLC cancer tissue and the adjacent normal tissue. Data were retrieved from Gene Expression Omnibus (GEO) dataset, with the accession number GSE29248. The color code in the heat map is linear and the expression levels of miRNAs that were upregulated are shown in green to red, whereas the miRNAs that were downregulated are shown from red to green. (B) The expression of miR-92a was further analyzed by qRT-PCR in 50 pairs of NSCLC tissue, along with 34 metastasis and 16 nonmetastatic NSCLC tissues. (C) Relative expression of miR-92a in non-metastatic $(n=16)$ and metastatic ( $\mathrm{n}=34)$ NSCLC samples. ${ }^{* *} \mathrm{P}<0.01$, compared with non-metastatic NSCLC samples. (D) Kaplan-Meier overall survival curve for NSCLC patients with miR-92a-high and miR-92a-low character. (E) Relative expression of miR-92a in four NSCLC cell lines (A549, H1299, SPC-A1 and H358) and a normal human bronchial epithelial cell line (16HBE). Data represent the means $\pm \mathrm{SD},{ }^{*} \mathrm{P}<0.05 ;{ }^{* *} \mathrm{P}<0.01$ vs. $16 \mathrm{HBE}$.

the cell monolayer was scraped using a $10 \mu \mathrm{l}$ micropipette tip, and then cultured with serum-free medium for $24 \mathrm{~h}$. The gap distances of migrating cells were calculated from photomicrographs as previously described (21).

Western blotting. Total cellular proteins were lysed in RIPA buffer in the presence of proteinase inhibitor (Sigma,
St. Louis, MO, USA). Concentrations of total cellular protein were determined using a BCA assay kit (Pierce, Rockford, IL, USA). Protein samples $(25 \mu \mathrm{g})$ were separated by $8 \%$ SDS-PAGE and then transferred onto polyvinylidene difluoride (PVDF) membranes (Millipore, Bedford, MA, USA). The membranes were incubated with primary antibodies at $4^{\circ} \mathrm{C}$ overnight using the following concentrations 
A

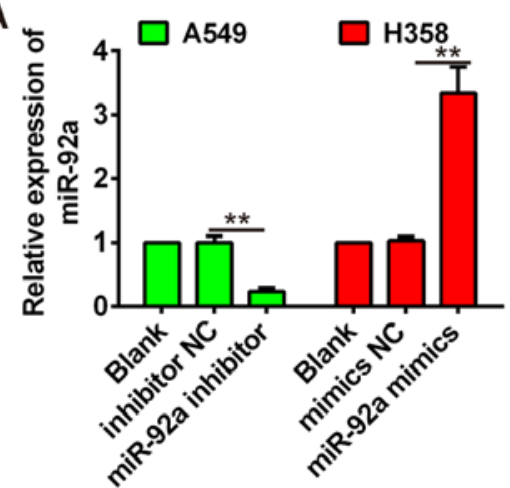

D
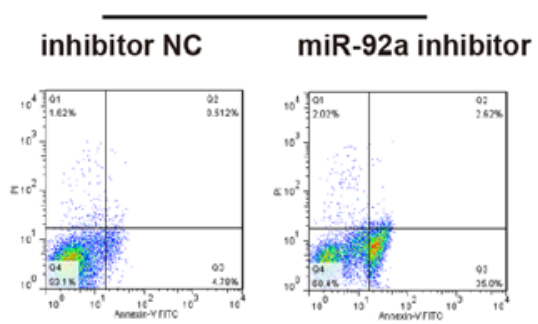

G

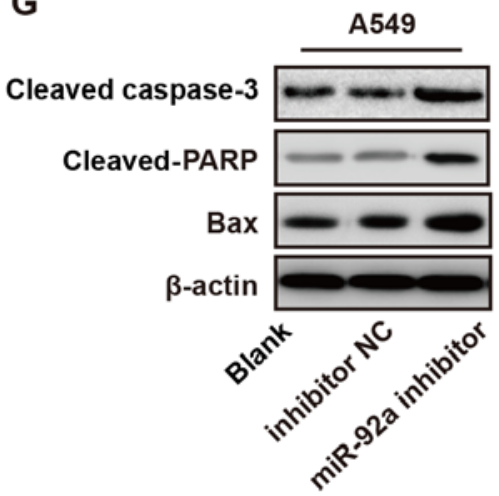

B

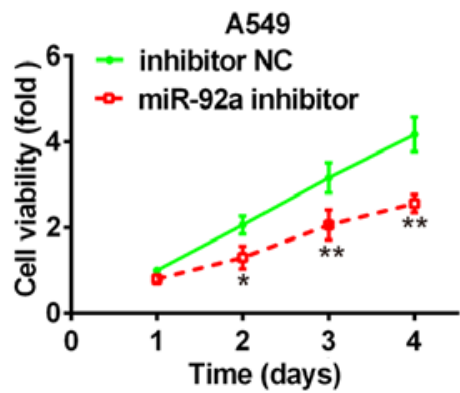

E

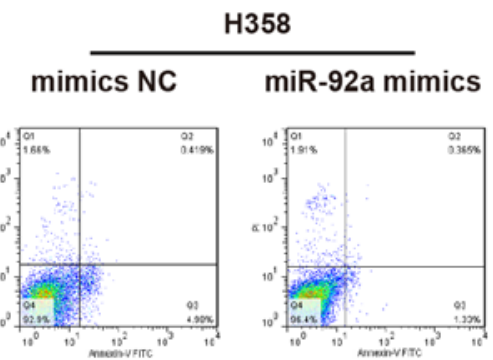

H

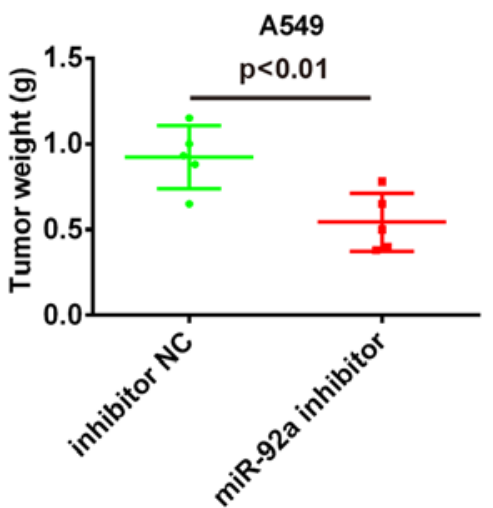

C

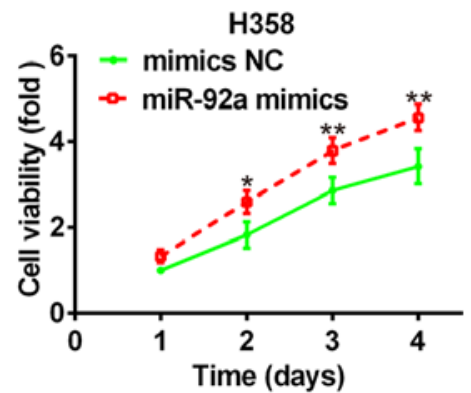

$\mathbf{F}$

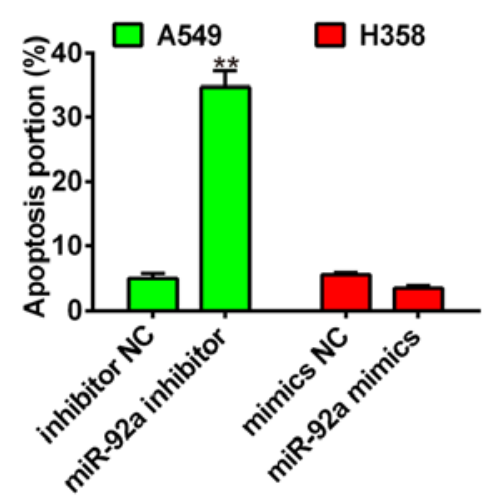

I

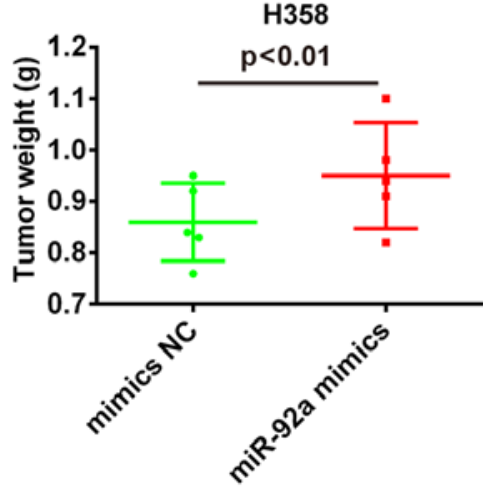

Figure 2. miR-92a promotes cell proliferation in vitro and tumor growth in vivo. (A) Transfection of miR-92a inhibitor significantly decreased the expression level of miR-92a in A549 cells, whereas increased in miR-92a mimic transfected H358 cells. (B and C) Cell proliferation was measured by MTT assay after transfection in A549 and H358 cells. (D-F) The percentage of apoptotic cells was evaluated by flow cytometry after transfection in A549 and H358 cells. (G) The expression levels of cleaved-caspase-3, cleaved-PARP and Bax were detected by western blotting after transfection in A549 and H358 cells. "P<0.05, ${ }^{* *} \mathrm{P}<0.01$ vs. negative control groups (inhibitor NC or mimic NC). (H and I) miR-92a inhibitor-transfected A549 cells and miR-92a mimic-transfected H358 cells were subcutaneously injected into the mouse right flank at $1 \times 10^{6}$ cells $/ 100 \mu l$ to establish NSCLC xenograft models, $\mathrm{n}=10$ per group. After 5 weeks of the transplantation, tumor-bearing mice were sacrificed and the xenografts were harvested and weighed. $\mathrm{P}<0.01$ vs. negative control groups (inhibitor NC or mimic NC).

of PTEN, $\beta$-catenin, Vimentin, N-cadherin, E-cadherin, AKT, phospho-mTOR and mTOR (1:1000; Cell Signaling Technology, Danvers, MA, USA), phospho-AKT (Ser473) (1:500; Cell Signaling Technology) and anti- $\beta$-actin (1:500; Santa Cruz Biotechnology, Santa Cruz, CA, USA), followed by horseradish peroxidase-conjugated secondary antibody (anti-rabbit, 1:2000, Cell Signaling Technology). Anti- $\beta$-actin antibody was used as an internal control. The detected protein signals were visualized using the ECL method (22).

Luciferase assays. The wild-type PTEN 3'-UTR and mutated PTEN 3'-UTR were amplified and cloned downstream of the luciferase gene in a pGL3 reporter plasmid (Promega). The constructed vectors were named as wt-PTEN-PGL3 and
mut-PTEN-PGL3, respectively. For the luciferase reporter assays, HEK 293 cells were cultured in 24-well plates, and each well was co-transfected with wt (mut)-PTEN-PGL3 and miR-control, miR-195 mimics or inhibitor and cultured for $48 \mathrm{~h}$. The luciferase activities were measured using the DualLuciferase Reporter Assay System (Promega, Madison, WI, USA), according to the manufacturer's protocol.

Tumor xenograft animal model. A549 cells transfected with miR-92a inhibitor or the inhibitor NC and H358 cells transfected with miR-92a mimic or mimic NC were subcutaneously injected into four-week-old male athymic nude mice at $3 \times 10^{6}$ cells in $0.2 \mathrm{ml}$ PBS per mouse, 5 mice per group. After 5 weeks, mice were sacrificed and tumor weight was detected. 
A

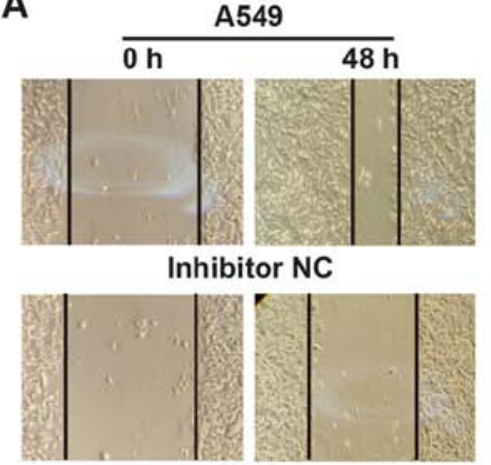

miR-92a inhibitor
B

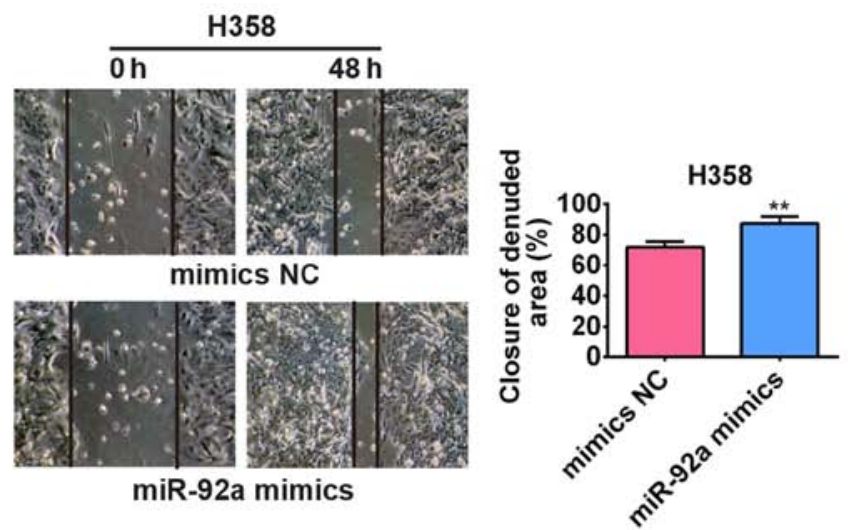

C
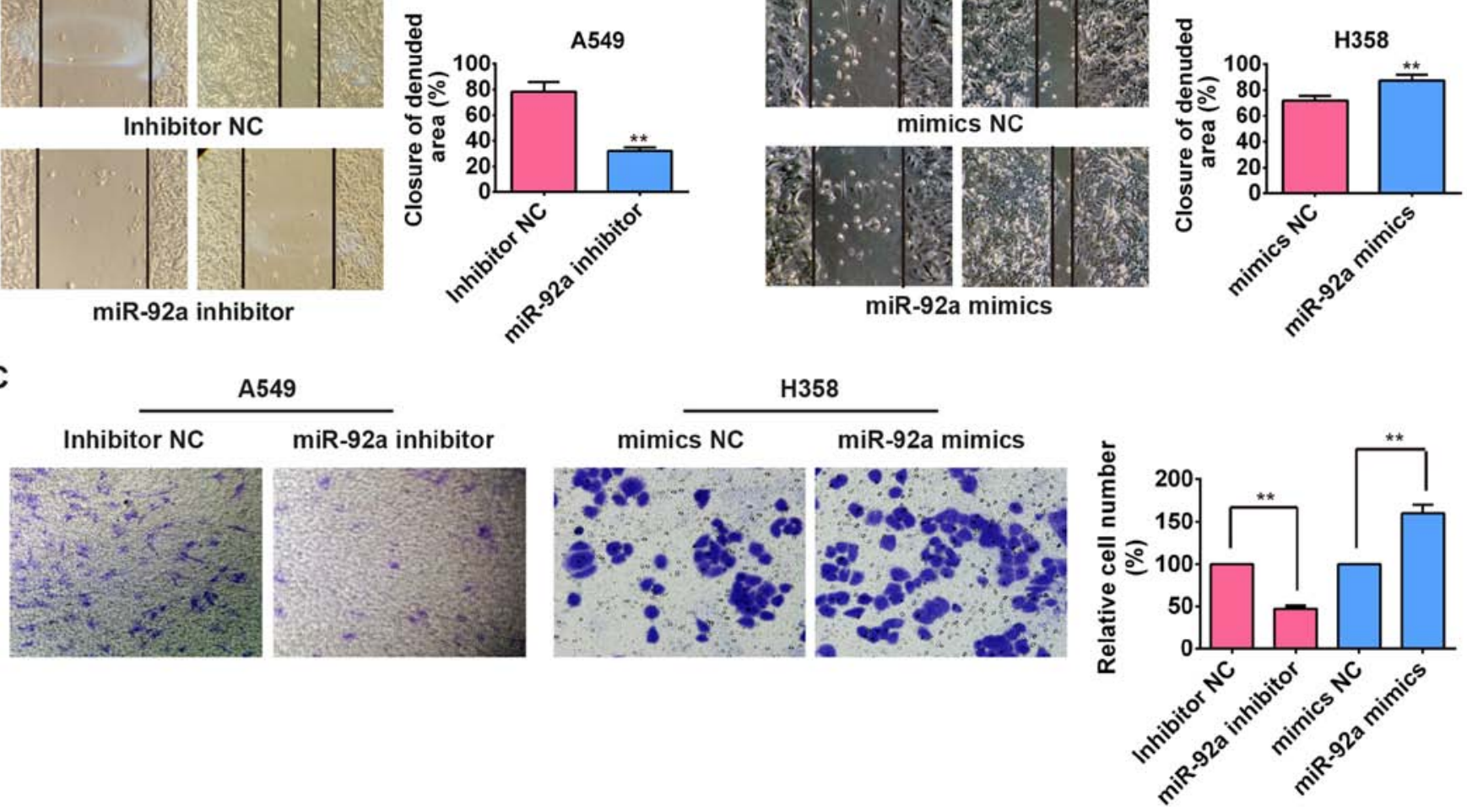

Figure 3. miR-92a promotes NSCLC cell metastasis. (A) Knockdown of miR-92a significantly inhibited the migration of A549 cells. (B) Overexpression of miR-92a significantly promoted the migration of $\mathrm{H} 358$ cells. (C) Knockdown of miR-92a significantly inhibited the invasion of A549 cells, while overexpression of miR-92a significantly promoted the invasion of $\mathrm{H} 358$ cells. ${ }^{* *} \mathrm{P}<0.01$ vs. negative vector control groups (inhibitor NC or mimic NC).

All experiments were performed in the Animal Institute of Second Military Medical University and approved by the Medical Experimental Animal Care Commission of Second Military Medical University.

Statistical analysis. Statistical analysis was performed with the GraphPad Prism 5.0 software. Data are reported as mean \pm SD. Statistical significance was calculated by Student's $\mathrm{t}$-test among different groups. Pearson's or Spearman's analysis was used in correlation analysis. A P-value $<0.05$ was considered to indicate a statistically significant difference.

\section{Results}

miR-92a is upregulated in both NSCLC cells and clinical specimens. To explore the role of miRNAs in NSCLC, we first analyzed the differential expressed miRNAs via retrieving the microarray data in the GEO dataset (GSE29248). Cluster analysis based on the miRNA expression pattern indicated a significant difference between NSCLC cancer tissue and the adjacent normal tissue (Fig. 1A). It is well known that EMT play a key role in the metastasis of tumors $(11,23)$, while miR-92a is reported to promote EMT progress in several other types of human cancer (24). For this reason, miR-92a was chosen as the candidate for further study.

To validate the expression of miR-92a obtained from miRNA microarray assay, qRT-PCR was applied to examine miR-92a expression in 50 pairs of NSCLC tissues, along with 34 metastasis and 16 non-metastatic NSCLC tissues. The result showed that miR-92a was significantly higher in NSCLC tissues than those of their matched adjacent normal tissues (Fig. 1B). miR-92a was also overexpressed in metastatic tissues compared with non-metastatic NSCLC tissues (Fig. 1C). To further investigate the correlation between miR-92a expression and prognosis of NSCLC patients, 50 patients were divided into 2 subgroups based on the mean of all samples: low miR-92a group $(\mathrm{n}=21)$ : miR-92a expression ratio < median ratio; high miR-92a group $(\mathrm{n}=29)$ : miR-92a expression ratio $>$ median ratio. From Kaplan-Meier survival curve, we observed that patients with high miR-92a expression had significantly shorter overall survival than those with low miR-92a expression ( $\mathrm{P}<0.001$, log-rank test; Fig. 1D).

To validate whether upregulation of miR-92a was also present in NSCLC cell lines, we examined miR-92a expression in four NSCLC cell lines including A549, H358, SPC-A1, H1299, and a normal human bronchial epithelial cell line $16 \mathrm{HBE}$ acted as a control. It was found that the expression of miR-92a was also aberrantly upregulated in four NSCLC cell lines, as compared to 16HBE (Fig. 1E), and these results were similar with the detection in NSCLC tissues. These results indicated that miR-92a may be involved in the development of NSCLC.

miR-92 a promotes cell proliferation in vitro and tumor growth in vivo. Given the upregulation of miR-92a in NSCLC tissues and cell lines, we predicted that miR-92a may function as a 
A

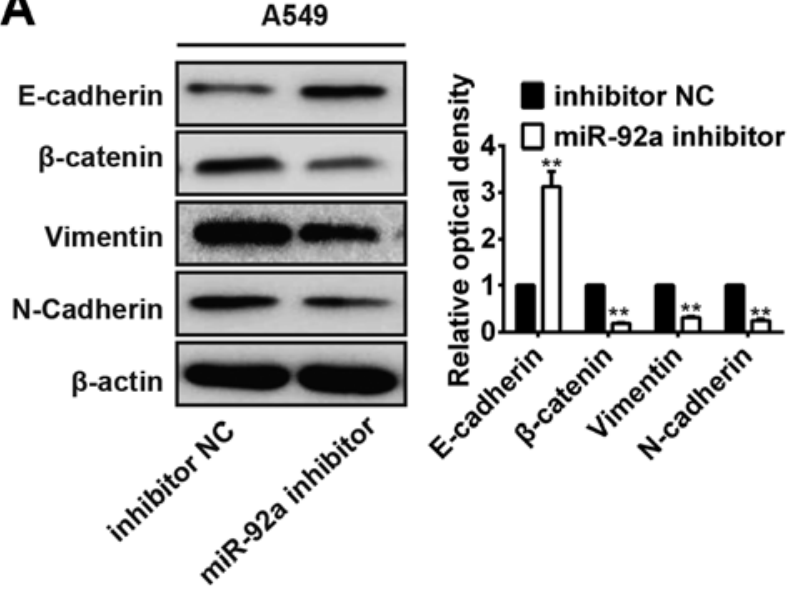

B

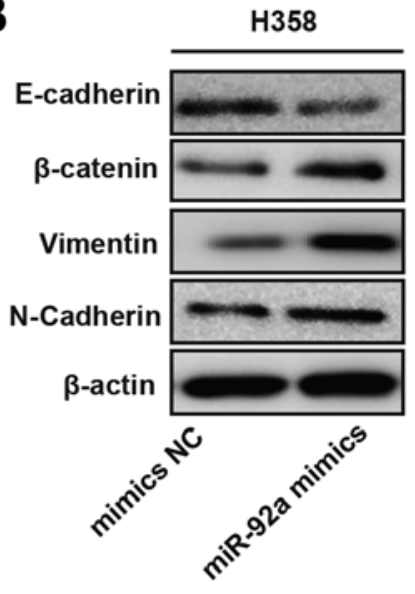

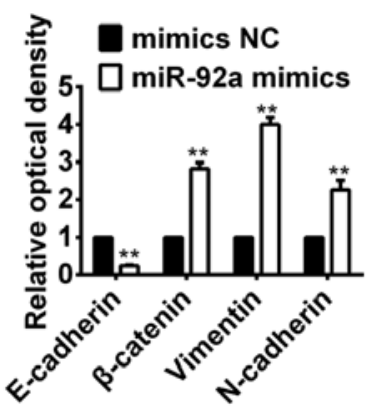

Figure 4. miR-92a promoted the EMT of NSCLC cells. (A) Expression levels of EMT markers including E-cadherin N-cadherin, vimentin and $\beta$-catenin were evaluated at protein levels in A549 cells transfected with miR-92a inhibitor. (B) Expression levels of EMT markers were evaluated at protein levels in $\mathrm{H} 358$ cells treated with miR-92a mimic. Data represent the means $\pm \mathrm{SD},{ }^{* *} \mathrm{P}<0.01$ vs. mimic NC or inhibitor NC.

tumor oncogene. To verify our hypothesis, miR-92a inhibitor was transfected into A549 cells and miR-92a mimic was transfected into H358 cells because A549 and H358 cells exhibited the highest and lowest expression levels of miR-92a in these NSCLC cell lines, respectively. qRT-PCR analysis showed that miR-92a expression was effectively reduced or enhanced by miR-92a inhibitor or mimic (Fig. 2A). The results of MTT revealed that miR-92a knockdown inhibited A549 cell proliferation, whereas miR-92a overexpression promoted H358 cell proliferation (Fig. 2B and C). Then, the percentage of apoptotic cells in miR-92a-transfected A549 and H358 cells was evaluated by flow cytometry. As shown in Fig. 2D-F, the ratio of apoptotic cells in A549 cells was increased, whereas decreased in $\mathrm{H} 358$ cells. To further study possible mechanisms through which miR-92a alters NSCLC cell apoptosis, we examined the expression of apoptosis-relevant proteins. As shown in Fig. 2G, miR-92a knockdown resulted in an obvious increased expression level of pro-apoptotic proteins including the cleaved-caspase-3, caspase-PARP and Bax in A549 cells.

To evaluate the effect of miR-92a on NSCLC tumor growth in vivo, A549 cells transfected with miR-92a inhibitor and H358 cells transfected with miR-92a mimic were subcutaneously injected into nude mice, and the growth of the resultant primary tumors were monitored. As expected, the tumor growth was significantly inhibited in miR-92a inhibitor transfected A549 cells (Fig. 2H), whereas significantly promoted in miR-92a mimic transfected H358 cells (Fig. 2I). All these results suggested that miR-92a executed an oncogenic effect in NSCLC by potentiating cell growth ability of NSCLC cells.

miR-92a promotes the migration and invasion of NSCLC cells in vitro. To explore whether miR-92a affects migration and invasion of NSCLC cells, wound healing and Transwell invasion assays were performed in A549 and H358 cells. Transfection of miR-92a inhibitor significantly attenuated the capacity of migration in A549 cells compared with the control cells without transfection (Fig. 3A), whereas the capacity of wound healing in $\mathrm{H} 358$ cells was significantly enhanced after treated with miR-92a mimic (Fig. 3B). Subsequently, the relative invaded cell number of A549 cells transfected with miR-92a inhibitor was significantly decreased compared with the control cells while the relative cell number was significantly increased in the miR-92a mimic transfected H358 cells (Fig. 3C), suggesting that miR-92a plays an important role in the regulation of NSCLC cellular motility, including the cell invasive and metastatic capacity.

miR-92a regulates EMT in NSCLC cells. Recent studies indicate that EMT plays an important role in the invasion and metastasis of NSCLC (25) and miRNAs have been recognized as key regulators of EMT (26), thus we assessed the ability of miR-92a to promote EMT in NSCLC cells. We found that the expression of E-cadherin known as the epithelial marker was significantly upregulated, whereas that of the mesenchymal marker $\mathrm{N}$-cadherin, vimentin and $\beta$-catenin were significantly downregulated in A549 cells transfected with miR-92a inhibitor (Fig. 4A). In contrast, overexpression of miR-92a markedly decreased E-cadherin expression and increased $\mathrm{N}$-cadherin, vimentin and $\beta$-catenin expression levels in miR-92a mimic transfected H358 cells (Fig. 4B). These results suggest that miR-92a controls NSCLC invasion and metastasis by regulating EMT.

PTEN is a direct target of miR-92a in NSCLC cells. To further characterize the molecular mechanisms involved in the oncogenic role of miR-92a in NSCLC cells, we searched for potential target genes of miR-92a using TargetScan and microRNA.org. PTEN was chosen as a target gene of miR-92a because its level is related with poor prognosis in lung cancer and facilitated EMT in colorectal cancer (CRC) (24,27-29). The predicted binding sites for miR-92a in the PTEN sequence are illustrated in Fig. 5A. Then, western blot analysis confirmed that overexpression of miR-92a markedly inhibited PTEN expression on protein level in H358 cells (Fig. 5B and D). In contrast, inhibition of miR-92a markedly promoted PTEN expression on protein level in A549 cells (Fig. 5C and D). Notably, overexpression of miR-92a in H358 cells or knockdown of miR-92a in A549 cells did not change 


\section{A}

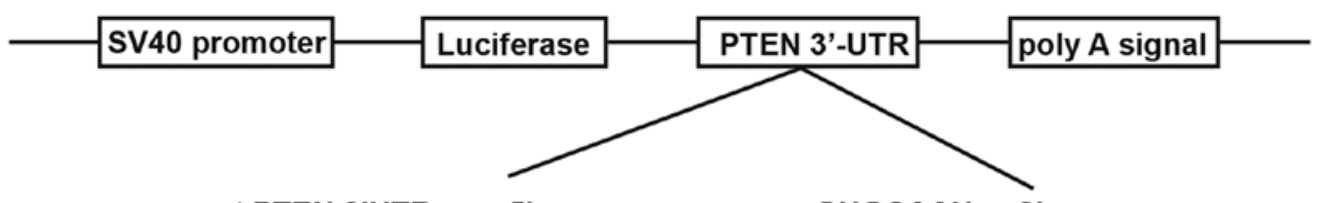

wt PTEN 3'UTR: 5' - guucuagaaauuuugUGCAAUa - 3'

hsa-miR-92a: 3'- uguccggcccuguuCACGUUAu - 5'

mut PTEN 3'UTR: 5' - guucuagaaauuuu EAAUGCA a - 3'

B
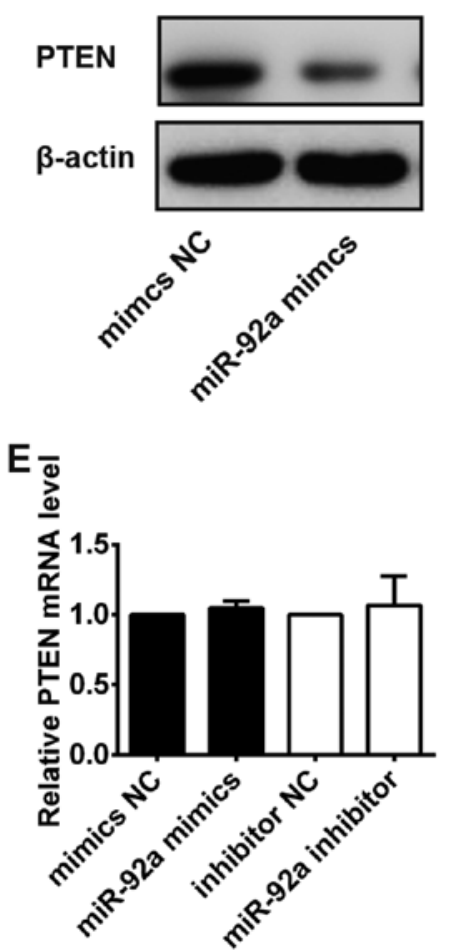

C

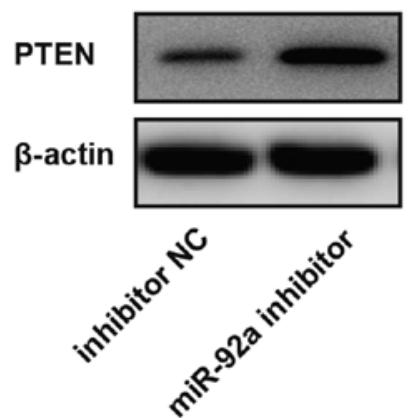

F

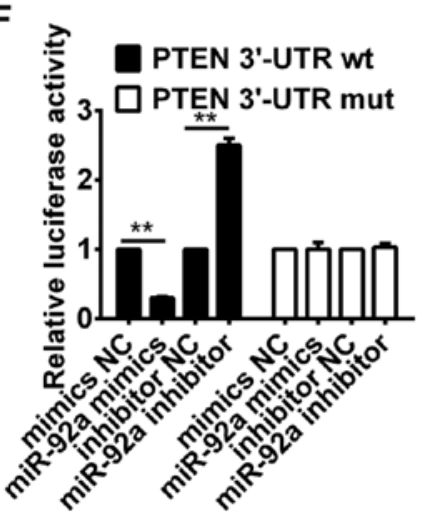

D

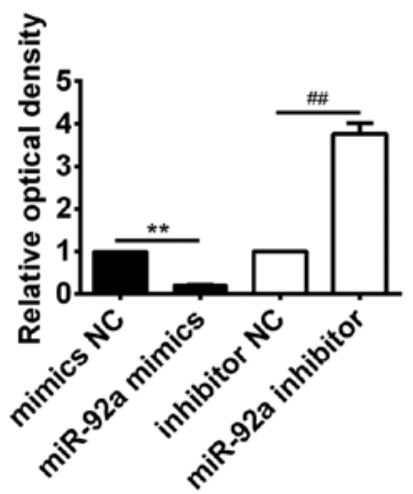

G

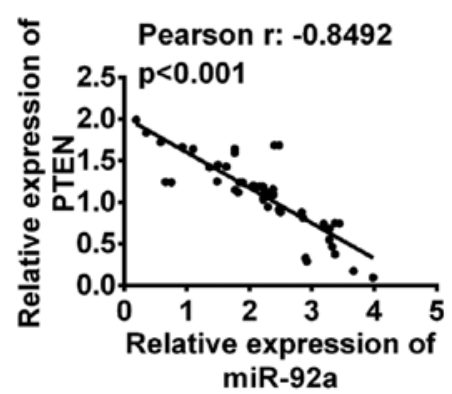

Figure 5. PTEN is a direct target of miR-92a in NSCLC cells. (A) The predicted complementary sequences for miR-92a in the 3'UTR of PTEN and the mutations are shown in the seed region of miR-92a. (B-D) PTEN protein expression was measured in A549 cells transfected with miR-92a inhibitor or in H358 cells treated with miR-92a mimic. $\beta$-actin was used as the internal control. Data represent the means $\pm \mathrm{SD},{ }^{\prime} \mathrm{P}<0.05,{ }^{* *} \mathrm{P}<0.01$ vs. miR-NC. (E) PTEN mRNA expression was measured in A549 cells transfected with miR-92a inhibitor or in H358 cells treated with miR-92a mimic. GAPDH was used as the internal control. Data represent the means $\pm \mathrm{SD},{ }^{*} \mathrm{P}<0.05,{ }^{* *} \mathrm{P}<0.01$ vs. miR-NC. (F) miR-92a mimic or inhibitor significantly inhibited or promoted the luciferase activity that carried wt 3'-UTR of PTEN but had no obvious effect on mt 3'-UTR of PTEN. (G) Pearson's analysis for the correlation of PTEN and miR-92a expression levels in patients with NSCLC $(n=50 ; r=-0.8492 ; \mathrm{P}<0.001)$.

A

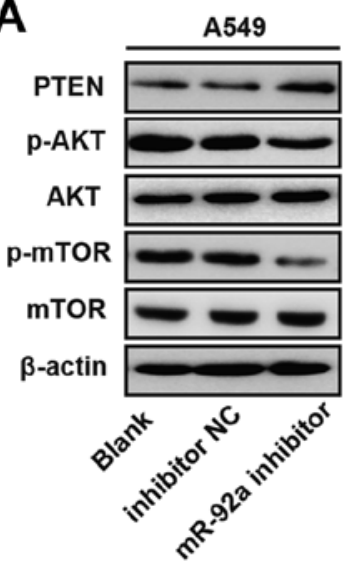

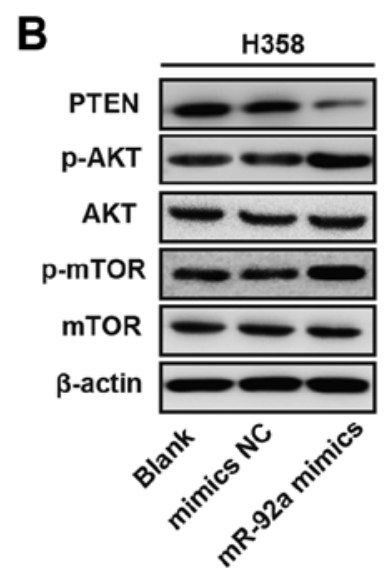

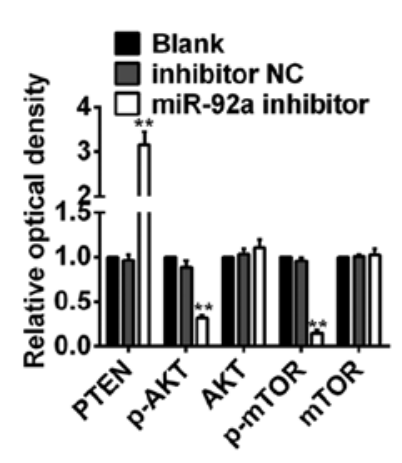

Figure 6. miR-92a regulates the PTEN-mediated AKT signal pathways. Protein levels of PTEN, p-AKT, AKT, p-mTOR and mTOR were detected by western blot analysis in miR-92a inhibitor transfected A549 cells (A) or in miR-92a mimic transfected H358 cells (B), and bands were semi-quantitatively analyzed by using ImageJ software, normalized to $\beta$-actin density. Data represent the means $\pm \mathrm{SD},{ }^{* *} \mathrm{P}<0.01$ vs. the blank and negative control groups. 


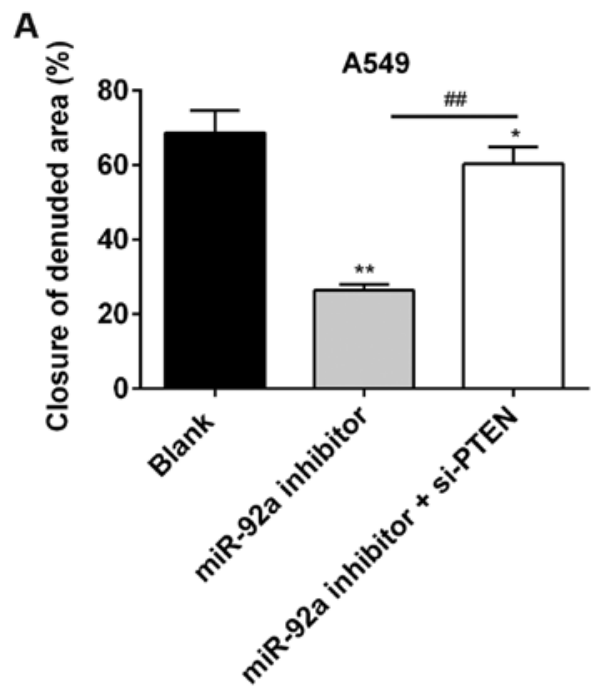

B
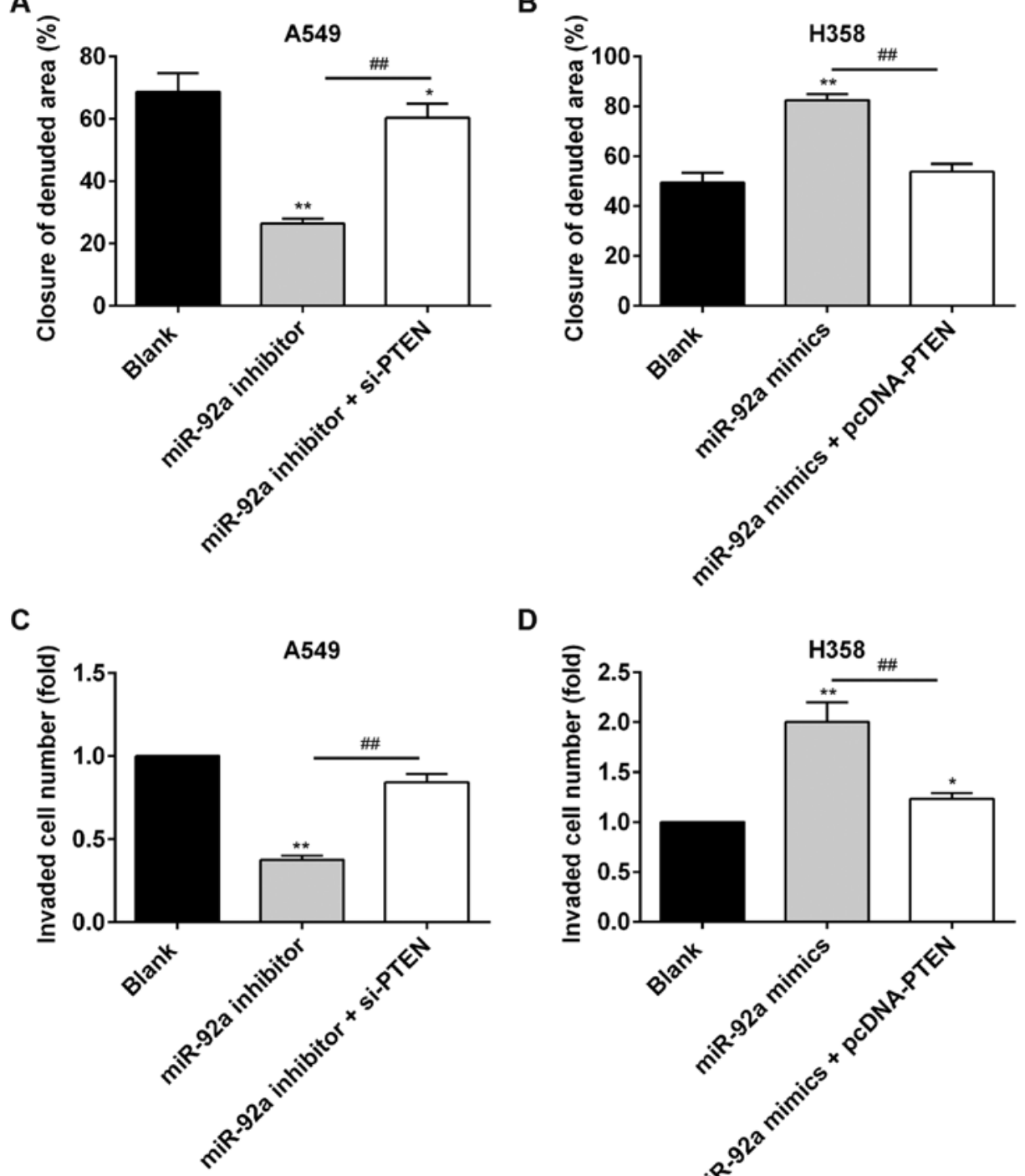

D

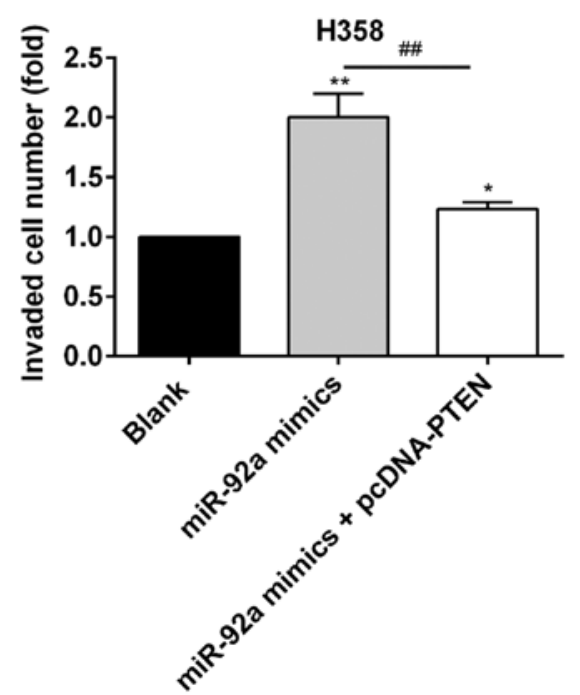

Figure 7. Repression of PTEN is required for the miR-92a-mediated promotion of cell migration and invasion in NSCLC. (A and C) Suppression of PTEN in A549 cells reversed the inhibitory effect of miR-92a inhibitor on cell migration and invasion. (B and D) Overexpression of PTEN in H358 cells attenuated the promoting effect of miR-92a mimic on cell migration and invasion. Data represent the means $\pm \mathrm{SD},{ }^{,} \mathrm{P}<0.05,{ }^{* *} \mathrm{P}<0.01$ vs. blank, ${ }^{\# \#} \mathrm{P}<0.01 \mathrm{vs}$. miR-92a inhibitor or miR-92a mimic.

PTEN mRNA level, which indicated miR-92a targeted PTEN mainly via translational inhibition (Fig. 5E). This finding agrees with the results reported by Zhang et al in colorectal cancer (24).

To determine whether PTEN is a direct target of miR-92a in NSCLC cells, luciferase reporter assay was performed. The result showed that overexpression of miR-92a significantly inhibited the luciferase activity of wild-type PTEN 3'UTR, while inhibition of miR-92a obviously promoted the luciferase activity of wild-type PTEN 3'UTR, but the activity of the mutant-type PTEN 3'UTR was not changed (Fig. 5F).

To further investigate the relationship between miR-92a and PTEN in NSCLC tissues, the level of PTEN mRNA in 50 NSCLC patients was measured by qRT-PCR (data not shown). As shown in Fig. 5G, Pearson's correlation analysis revealed that the expression of miR-92a was inversely correlated with the expression of PTEN in the 50 patients with NSCLC $(r=-0.8492 ; \mathrm{P}<0.001)$. These results indicated that
miR-92a could directly bind anti-oncogenic PTEN and inhibited its expression in NSCLC.

miR-92a activates the PTEN mediated AKT signal pathway. It is reported that PTEN can negatively regulate the activity of Akt $(30,31)$, and the PI3K/Akt pathway plays an important role in the invasion and metastasis of tumor cells (32-34). However, the effect of miR-92a on PTEN-mediated AKT signaling was not reported previously in NSCLC. Western blot analysis revealed that miR-92a knockdown in A549 cells robustly increased PTEN protein expression levels, inhibited the phosphorylated AKT (Ser 473) and phosphorylated-mTOR (Fig. 6A), whereas the inhibition of miR-92a in H358 cells exerted the opposite effect (Fig. 6B). However, miR-92a overexpression or miR-92a knockdown did not change the expression levels of total AKT and mTOR protein (Fig. 6A and B). These results indicate that miR-92a promotes the activity of PI3K/AKT pathway in NSCLC cells. 
PTEN is required for the $\mathrm{miR}-92 a$-mediated promotion of cell migration and invasion in NSCLC. To evaluate if PTEN is responsible for the oncogenic potential of miR-92a in NSCLC cells, gain- and loss-of-function studies of PTEN were performed. As expected, PTEN silencing by si-PTEN partially reversed the effects of miR-92a knockdown on cell migration and invasion in A549 cells (Fig. 7A and C). Conversely, overexpression of PTEN significantly alleviated the enhancement of cell migration and invasion induced by miR-92a mimic in H358 cells (Fig. 7B and D). Together, these data suggest that miR-92a exerts an oncogenic role by targeting PTEN/PI3K/ AKT pathway in NSCLC.

\section{Discussion}

In the present study, we demonstrated that miR-92a is frequently upregulated in human NSCLC tissues and cell lines, and significantly correlated with poor prognosis. Furthermore, miR-92a can regulate NSCLC cellular metastasis by inducing EMT through activating PI3K/AKT pathways, at least partially by downregulating the level of PTEN. These results suggest that miR-92a functions as an oncogene in NSCLC and may serve as a novel and promising therapeutic target for NSCLC.

Increasing evidence has demonstrated that miRNAs play a crucial role in NSCLC invasion and metastasis $(35,36)$. Some miRNAs, for instance miR-663a, miR-361-3p and miR491-5p, have been proved to contribute to the metastasis of NSCLC (37-39). Recent studies showed that the expression of miR-92a was increased in NSCLC tissues, and overexpression of miR-92a in NSCLC cells promoted growth, metastasis, and chemoresistance by targeting PTEN (40). Noteworthy, miR-92a was found upregulated in human NSCLC tissues and cell lines via retrieving the microarray data in the GEO dataset (GSE29248), which was consistent with previous results (24). Thus, we chose miR-92a for further study. Moreover, the in vitro and in vivo assay results confirmed that miR-92a could promote proliferation, migration in NSCLC cells and tumor formation in nude mice. In addition, we found that upregulation of miR-92a was significantly correlated with shorter overall survival. These results suggested that miR-92a plays an oncogenic role in NSCLC.

EMT is a crucial event in tumor migration and metastasis, which is the first indication of cancer development (41). MicroRNAs recently emerged as important regulators of EMT in various cancers $(26,42)$. For example, the miR-221/222 miRNA cluster has been found to induce EMT in breast cancer cells (43). miR-27 is upregulated in gastric cancer metastasis and enhances EMT through regulation of Zeb1, Zeb2 and Slug (44). These studies support a crucial role of microRNAs in controlling EMT and metastasis. For NSCLC, the role of miRNAs in EMT is being explored. Our results showed that the inhibition of miR-92a could suppress EMT of NSCLC cells whereas overexpression of miR-92a promoted EMT of NSCLC cells. Thus, our findings implicate miR-92a promote the metastasis of NSCLC cells by regulating EMT phenotype.

PTEN, a tumor suppressor, is a well-known regulator of EMT and inhibits tumor cell growth and invasion by blocking the PI3K/Akt pathway (45-48). In NSCLC, PTEN was found to be upregulated and associated with the metastatic phenotype of
NSCLC cells (29). In this study, our results showed that miR-92a directly targeted PTEN in NSCLC cells and the expression of PTEN was inversely correlated with miR-92 expression in NSCLC tissues. Furthermore, we found that upregulation of miR-92a could promote activation of PI3K/AKT signaling pathway through downregulation of PTEN. Of note, overexpression of PTEN effectively inhibited the tumor promotional functions of miR-92a on NSCLC migration and invasion.

In conclusion, our results reinforce the role of miR-92a as a tumor oncogene in NSCLC. Importantly, we identified a likely novel potential mechanism of miR-92a promoting EMT and tumor metastasis by inhibiting PTEN and indirectly regulating PI3K/AKT signaling pathway. In particular, the important role of miR-92a in NSCLC suggests that the upregulation of miR-92a may have diagnostic and therapeutic value for NSCLC.

\section{References}

1. Spira A and Ettinger DS: Multidisciplinary management of lung cancer. N Engl J Med 350: 379-392, 2004

2. Mercier O, Fadel E, de Perrot M, Mussot S, Stella F, Chapelier A and Dartevelle P: Surgical treatment of solitary adrenal metastasis from non-small cell lung cancer. J Thorac Cardiovasc Surg 130: 136-140, 2005.

3. Fabian MR, Sonenberg N and Filipowicz W: Regulation of mRNA translation and stability by microRNAs. Annu Rev Biochem 79: 351-379, 2010.

4. Guo H, Ingolia NT, Weissman JS and Bartel DP: Mammalian microRNAs predominantly act to decrease target mRNA levels. Nature 466: 835-840, 2010.

5. Bartel DP: MicroRNAs: Genomics, biogenesis, mechanism, and function. Cell 116: 281-297, 2004.

6. Boeri M, Sestini S, Fortunato O, Verri C, Suatoni P, Pastorino U and Sozzi G: Recent advances of microRNA-based molecular diagnostics to reduce false-positive lung cancer imaging. Expert Rev Mol Diagn 15: 801-813, 2015.

7. Skrzypski M, Dziadziuszko R and Jassem J: MicroRNA in lung cancer diagnostics and treatment. Mutat Res 717: 25-31, 2011.

8. Guan P, Yin Z, Li X, Wu W and Zhou B: Meta-analysis of human lung cancer microRNA expression profiling studies comparing cancer tissues with normal tissues. J Exp Clin Cancer Res 31: 54, 2012.

9. Shang Y, Zang A, Li J, Jia Y, Li X, Zhang L, Huo R, Yang J, Feng J, Ge K, et al: MicroRNA-383 is a tumor suppressor and potential prognostic biomarker in human non-small cell lung cancer. Biomed Pharmacother 83: 1175-1181, 2016.

10. Zeng Y, Zhu J, Shen D, Qin H, Lei Z, Li W, Liu Z and Huang JA: MicroRNA-205 targets SMAD4 in non-small cell lung cancer and promotes lung cancer cell growth in vitro and in vivo. Oncotarget: Jun 30, 2016 (Epub ahead of print).

11. Thiery JP, Acloque H, Huang RY and Nieto MA: Epithelialmesenchymal transitions in development and disease. Cell 139: 871-890, 2009.

12. Mahmood MQ, Ward C, Muller HK, Sohal SS and Walters EH: Epithelial mesenchymal transition (EMT) and non-small cell lung cancer (NSCLC): A mutual association with airway disease. Med Oncol 34: 45, 2017.

13. Qin Q, Wei F, Zhang J and Li B: miR-134 suppresses the migration and invasion of non-small cell lung cancer by targeting ITGB1. Oncol Rep 37: 823-830, 2017.

14. Lamouille S, Xu J and Derynck R: Molecular mechanisms of epithelial-mesenchymal transition. Nat Rev Mol Cell Biol 15: 178-196, 2014.

15. Hao J, Zhang Y, Deng M, Ye R, Zhao S, Wang Y, Li J and Zhao Z: MicroRNA control of epithelial-mesenchymal transition in cancer stem cells. Int J Cancer 135: 1019-1027, 2014.

16. Yu M, Xue H, Wang Y, Shen Q, Jiang Q, Zhang X, Li K, Jia M, Jia J, Xu J, et al: miR-345 inhibits tumor metastasis and EMT by targeting IRF1-mediated mTOR/STAT3/AKT pathway in hepatocellular carcinoma. Int J Oncol 50: 975-983, 2017.

17. Sathyanarayanan A, Chandrasekaran KS and Karunagaran D: microRNA-145 modulates epithelial-mesenchymal transition and suppresses proliferation, migration and invasion by targeting SIP1 in human cervical cancer cells. Cell Oncol (Dordr) 40: 119-131, 2017. 
18. Korpal M,Lee ES,Hu G and Kang Y: The miR-200 family inhibits epithelial-mesenchymal transition and cancer cell migration by direct targeting of E-cadherin transcriptional repressors ZEB1 and ZEB2. J Biol Chem 283: 14910-14914, 2008.

19. Korpal M and Kang Y: The emerging role of miR-200 family of microRNAs in epithelial-mesenchymal transition and cancer metastasis. RNA Biol 5: 115-119, 2008.

20. Song CL, Liu B, Shi YF, Liu N, Yan YY, Zhang JC, Xue X, Wang JP, Zhao Z, Liu JG, et al: MicroRNA-130a alleviates human coronary artery endothelial cell injury and inflammatory responses by targeting PTEN via activating PI3K/Akt/eNOS signaling pathway. Oncotarget 7: 71922-71936, 2016.

21. Fukumoto I, Kinoshita T, Hanazawa T, Kikkawa N, Chiyomaru T, Enokida H, Yamamoto N, Goto Y, Nishikawa R, Nakagawa M, et al: Identification of tumour suppressive microRNA-451a in hypopharyngeal squamous cell carcinoma based on microRNA expression signature. Br J Cancer 111: 386-394, 2014.

22. da Silva Xavier G, Leclerc I, Varadi A, Tsuboi T, Moule SK and Rutter GA: Role for AMP-activated protein kinase in glucosestimulated insulin secretion and preproinsulin gene expression. Biochem J 371: 761-774, 2003.

23. Zheng $\mathrm{H}$ and Kang Y: Multilayer control of the EMT master regulators. Oncogene 33: 1755-1763, 2014.

24. Zhang G, Zhou H, Xiao H, Liu Z, Tian H and Zhou T: MicroRNA-92a functions as an oncogene in colorectal cancer by targeting PTEN. Dig Dis Sci 59: 98-107, 2014.

25. Zhang JX,Zhai JF, Yang XT and Wang J: MicroRNA-132 inhibits migration, invasion and epithelial-mesenchymal transition by regulating TGF $\beta 1 / \mathrm{Smad} 2$ in human non-small cell lung cancer. Eur Rev Med Pharmacol Sci 20: 3793-3801, 2016.

26. Lamouille S, Subramanyam D, Blelloch R and Derynck R: Regulation of epithelial-mesenchymal and mesenchymalepithelial transitions by microRNAs. Curr Opin Cell Biol 25 200-207, 2013.

27. Xiao J, Hu CP, He BX, Chen X, Lu XX, Xie MX, Li W, He SY, You SJ and Chen Q: PTEN expression is a prognostic marker for patients with non-small cell lung cancer: A systematic review and meta-analysis of the literature. Oncotarget 7: 57832-57840, 2016.

28. Gu J, Ou W, Huang L, Wu J, Li S, Xu J, Feng J, Liu B and Zhou Y: PTEN expression is associated with the outcome of lung cancer: Evidence from a meta-analysis. Minerva Med 107: 342-351, 2016

29. Zhu DY, Li XN, Qi Y, Liu DL, Yang Y, Zhao J, Zhang CY, Wu K and Zhao S: MiR-454 promotes the progression of human non-small cell lung cancer and directly targets PTEN. Biomed Pharmacother 81: 79-85, 2016.

30. Chetram MA and Hinton CV: PTEN regulation of ERK1/2 signaling in cancer. J Recept Signal Transduct Res 32: 190-195, 2012.

31. Feng X, Jiang J, Shi S, Xie H, Zhou L and Zheng S: Knockdown of miR-25 increases the sensitivity of liver cancer stem cells to TRAIL-induced apoptosis via PTEN/PI3K/Akt/Bad signaling pathway. Int J Oncol 49: 2600-2610, 2016.

32. Xia H, Li Y and Lv X: MicroRNA-107 inhibits tumor growth and metastasis by targeting the BDNF-mediated PI3K/AKT pathway in human non-small lung cancer. Int J Oncol 49: 1325-1333, 2016.
33. Wang R, Zhang Q, Peng X, Zhou C, Zhong Y, Chen X, Qiu Y, Jin M, Gong M and Kong D: Stellettin B induces G1 arrest, apoptosis and autophagy in human non-small cell lung cancer A549 cells via blocking PI3K/Akt/mTOR pathway. Sci Rep 6: 27071, 2016.

34. Larue L and Bellacosa A: Epithelial-mesenchymal transition in development and cancer: Role of phosphatidylinositol 3' kinase/ AKT pathways. Oncogene 24: 7443-7454, 2005.

35. Xiao L, Zhou H, Li XP, Chen J, Fang C, Mao CX, Cui JJ, Zhang W, Zhou HH, Yin JY, et al: MicroRNA-138 acts as a tumor suppressor in non small cell lung cancer via targeting YAP1. Oncotarget 7: 40038-40046, 2016.

36. Pastorkova Z, Skarda J and Andel J: The role of microRNA in metastatic processes of non-small cell lung carcinoma. Biomed Pap Med Fac Univ Palacky Olomouc Czech Repub 160: 343-357, 2016.

37. Zhang Y, Xu X, Zhang M, Wang X, Bai X, Li H, Kan L, Zhou Y, Niu $\mathrm{H}$ and He P: MicroRNA-663a is downregulated in non-small cell lung cancer and inhibits proliferation and invasion by targeting JunD. BMC Cancer 16: 315, 2016.

38. Chen W, Wang J, Liu S, Wang S, Cheng Y, Zhou W, Duan C and Zhang C: MicroRNA-361-3p suppresses tumor cell proliferation and metastasis by directly targeting SH2B1 in NSCLC. J Exp Clin Cancer Res 35: 76, 2016.

39. Gong F, Ren P, Zhang Y, Jiang J and Zhang H: MicroRNAs491-5p suppresses cell proliferation and invasion by inhibiting IGF2BP1 in non-small cell lung cancer. Am J Transl Res 8: 485-495, 2016.

40. Ren P, Gong F, Zhang Y, Jiang J and Zhang H: MicroRNA-92a promotes growth, metastasis, and chemoresistance in non-small cell lung cancer cells by targeting PTEN. Tumour Biol 37: 3215-3225, 2016.

41. Hanahan D and Weinberg RA: Hallmarks of cancer: The next generation. Cell 144: 646-674, 2011.

42. Zaravinos A: The regulatory role of microRNAs in EMT and cancer. J Oncol 2015: 865816, 2015.

43. Stinson S, Lackner MR, Adai AT, Yu N, Kim HJ, O'Brien C, Spoerke J, Jhunjhunwala S, Boyd Z, Januario $\mathrm{T}$, et al: miR-221/222 targeting of trichorhinophalangeal 1 (TRPS1) promotes epithelial-to-mesenchymal transition in breast cancer. Sci Signal 4: pt5, 2011.

44. Zhang Z, Liu S, Shi R and Zhao G: miR-27 promotes human gastric cancer cell metastasis by inducing epithelial-tomesenchymal transition. Cancer Genet 204: 486-491, 2011.

45. Castellino RC and Durden DL: Mechanisms of disease: The PI3K-Akt-PTEN signaling node - an intercept point for the control of angiogenesis in brain tumors. Nat Clin Pract Neurol 3: 682-693, 2007.

46. Grille SJ, Bellacosa A, Upson J, Klein-Szanto AJ, van Roy F, Lee-Kwon W, Donowitz M, Tsichlis PN and Larue L: The protein kinase Akt induces epithelial mesenchymal transition and promotes enhanced motility and invasiveness of squamous cell carcinoma lines. Cancer Res 63: 2172-2178, 2003.

47. Vogt PK, Gymnopoulos M and Hart JR: PI 3-kinase and cancer: Changing accents. Curr Opin Genet Dev 19: 12-17, 2009.

48. Tu K, Liu Z, Yao B, Han S and Yang W: MicroRNA-519a promotes tumor growth by targeting PTEN/PI3K/AKT signaling in hepatocellular carcinoma. Int J Oncol 48: 965-974, 2016. 